\title{
SYMMETRIC SKEW 3-REVERSE DERIVATIONS WITH SEMIPRIME RINGS
}

\section{JAYA SUBBA REDDY ${ }^{1}$, K.CHENNAKESAVULU ${ }^{2} \&$ KOTTE AMARANADHA REDDY ${ }^{3}$}

${ }^{1}$ Associate Professor, Sri Venkateswara University, Tirupati, Andhra Pradesh

${ }^{2} S$. K. College of Agricultural Sciences, Anantapuramu, Andhra Pradesh

${ }^{3}$ Research Scholar, V.I.T, Vellore

\begin{abstract}
Suppose $\alpha$ be an anti automorphism in $R, \quad D: R \times R \times R \times \rightarrow R$ is a symmetric skew 3-reverse derivation with anti automorphism $\alpha$. $f$ trace in $D$ then $D(f(l), l, l)=0$, every $l \in I$, implies $D=0$.

KEYWORDS: Reverse Derivation and Anti automorphism.
\end{abstract}

Received: Feb 26, 2021; Accepted: Mar 16, 2021; Published: Jul 10, 2021; Paper Id.: IJESRJUN202116

\section{INTRODUCTION}

Faiza Shujat and Abuzaid Ansari [4] has studied symmetric skew 3-derivations on prime and semiprime rings. This paper proved that in semi prime ring with a non zero symmetric skew 3-reverse derivation is commutative.

\section{Preliminaries}

In this paper, If 2,3 - torsion free semiprime $\operatorname{ring} R$ with center $Z, \alpha$ is anti automorphism in $R$.The notaion $[x, y]$ is commutator $x y-y x$. Semi prime if $x R x=0$, then $x=0$.

Before starting our main theorem, using some results.

Let $D$ be a symmetric then

$$
D(-l, m, n)=-D(l, m, n) \text {, all in } R \text {. }
$$

\section{Theorem 1}

$D: R^{3} \rightarrow R$ is symmetric skew 3-reverse derivation in $R$ on $f$ is $D(f(l), l, l)=0$, all in $R$. Implies $D=0$.

\section{Proof}

Assume $D(f(l), l, l)=0, l$ in $R$.

Linearization of (2) and replace $m$ by $-m$, substitute $m+n$ for $m$, replacing $n$ by $w n$ and using symmetric in $D$, we have

$$
\begin{aligned}
& \quad 6 \alpha(D(m, m, n)) D(w, l, l)+6 \alpha(D(m, m, w)) D(\alpha(n), l, l)+24 \alpha(D(m, n, l)) D(w, l, m)+ \\
& 24 \alpha(D(m, w, l)) D(\alpha(n), l, m)+6 \alpha(D(l, l, n)) D(w, n, n)+6 \alpha(D(l, l, w)) D(\alpha(n), m, m)=0, \quad \text { for } \\
& l, m, n, w \in R .
\end{aligned}
$$


$D(l, l, n) D(w, l, l)=0$, any in $R$.

Substitute $w$ by $u w$ equation (4) and using equation (4) obtain

$D(l, l, n) u D(w, l, l)=0$

Semi prime ness, $D(w, l, l)=0$, for all $w, l \in R$.

By linearization implies $D(w, l, m)=0$, any $w, l, m \in R$.

Implies $D=0$.

\section{REFERENCES}

1. AjdaFosner.: Prime and semiprime rings with symmetric skew 3-derivations, Aequat.Math.87 (2014), 191-200.

2. C. Jaya Subba Reddy, V. Vijaya Kumar \& K. Hemavathi, "Prime Ring with Symmetric Skew 3 - Reverse Derivation", International Journal of Mathematics and Computer Applications Research (IJMCAR), Vol. 4, Issue 6, $p p, 69-74$

3. Argac, N.: On prime and semiprime rings with derivations. Algebra Colloq. 13(2006),371-380.

4. K. Chennakesavulu, C. Jaya Subba Reddy, A. Siva Kameswara Kumar \& K. Nagesh, "The Commutativity of Rings With Left Multiplicative Generalized Derivations", International Journal of Educational Science and Research (IJESR), Vol. 8, Issue 6, pp, 53-64

5. Bresar.M. and Vukman.J.: On some additive mappings in rings with involution, Aequationes Math.38(1989),178185.

6. FaizaShujatandAbuzaid Ansari.: Symmetric skew 4-derivations on prime rings, J. Math. Comput. Sci. 4 (2014), No.4, 649-656

7. Sharma Poonam, Kaur Kamaldeep, Chawla Amit, Singh Ranjodh, \& Singh Rahuldev Dhawan R.K, "A Review on Biological Activities of Quinoline Derivatives", BEST: Journal of Management, Information Technology and Engineering (BEST: JMITE) ,Vol. 2, Issue 1, pp, 1-14

8. Jaya SubbaReddy.C et al.: prime ring with symmetric skew 3-reverse derivations, International Journal of Mathematics and Computer Applications Research, Vol. 4, Issue 6,(2014), 69-74.

9. Revathi Pandian, “A Study on Financial Derivatives (Futures \& Options)”, IMPACT: International Journal of Research in Business Management (IMPACT: IJRBM), Vol. 3, Issue 3, pp, 1-15

10. Jung, Y.S, Park, K.H.: On prime and semiprime rings with permuting 3-derivations.Bull.Korean Math.Soc.44(2007),789-794.

11. Park, K.H.: On prime and semiprime rings with permuting n-derivations.J.ChungcheongMath.Soc.22(2009),451458.

12. Posner,E.C.: Derivations in prime rings.Proc.Am.Math.Soc.8(1957),1093-1100. 
13. Samman.M. and Alyamani. N.: Derivations and reverse derivations in semi prime rings. International Mathematical Fourm, 2, no.39(2007), 1895-1902.

14. J. Vukman.: Symmetric biderivations on prime and semiprime rings, Aequationes Math.38(1989), 245-254.

15. KotteAmaranadha Reddy et al.: Review on Orthogonal Derivation in Rings, Research Journal of pharmacy and Technology, 12, no 4 (2019), 1991-1996.

16. J. Vukman.:Two results concerning symmetric biderivations on prime rings, Aequationes Math. 40 (1990), 181189. 
